\title{
Primary Health Care
}

\section{Parvin $\mathbf{R}^{*}$}

Department of Medicine, Enam Medical College and Hospital, Savar, Dhaka, Bangladesh

Primary health care (PHC) is regarded as the first element of a continuing health care process. It acts as a bridge between community and national health system. According to World Health Organization (WHO) Alma-Ata declaration of 1978, primary health care is defined as, 'Essential health care based on practical, scientifically sound and socially acceptable methods and technology', made universally accessible to individuals and families in the community through their full participation, and at a cost that the community and country can afford to maintain at every stage of their development, in the spirit of self-reliance and self-determination [1]. Primary health care should be the central focus of a health care system. Prevention and development of complication of several diseases can be achieved if proper primary care can be provided. Therefore, it should be readily available and easily accessible to all individuals, irrespective to gender, age, caste, color, urban or rural location and social class [2].

The concept of PHC was influenced by the experience of the medical missionaries working in the developed countries, who helped in training of the village workers at the grassroots level. PHC also got inspirations from the Chinese 'Barefoot doctors', who were globally popular for their extensive health service at the rural areas. They provided combined western and traditional medicine, aimed to prevention of diseases rather than cure [2].

There were three key ideas which permeated the declaration 'appropriate technology'. First issue was criticism of negative role of 'disease-oriented technology'. It denoted that the modern instruments and machines are too sophisticated and expensive for the poor community. If the technology is not accessible, affordable, feasible and culturally acceptable, then the large portion of the population would remain out of health service. So, more health institutions should be established in the rural areas with the easily available and appropriate health tools, instead of urban hospital construction. Second issue was disapproval of the overspecialization of the health personnel in the developed countries. Emphasis was put upon the training of lay health personnel, including shamans and midwives. Community participation was stressed. Final issue was the link between health and development. Health work should be regarded as a way of development, which would improve living condition [3].

Infrastructure of primary health care is poorly developed in many countries. Teamwork is little, and services are not smooth. There is lack of communication between primary and secondary health care. Thus, there is increased reliance on specialist service and the hospitals, which can be reduced by appropriate primary care. To deliver universal health coverage, developing countries are trying to adopt team-based approach. PHC is cost effective, and it can help in a country's health strategy by reducing the disease burden, producing economic savings and assuring greater equity [4].

Primary health care approach was too broad and not realistic at the beginning. The idea of selective primary health care (SPHC) was proposed thereafter by Julia Walsh and Kenneth S. Warren, who presented a paper entitled 'Selective Primary Health Care, an Interim Strategy for Disease Control in Developing Countries', in a conference organized by Rockefeller foundation in Italy in 1979. They suggested targeting diseases, which cause greatest mortality and morbidity. New strategies were adopted, focusing the maternal and child health as part of primary care. Subsequently, the acronym GOBI-FFF appeared which included growth monitoring, oral rehydration therapy, breastfeeding, immunization, family planning, female education and food supplementation. In 2008, an assessment of progression of primary health care was done among 30 low-income and middleincome countries [5]. It revealed that these countries scaled up SPHC like immunization, family planning, etc. Fourteen countries progressed to comprehensive primary health care, which was marked by high coverage of skilled attendance at birth. Still, SPHC was criticized for having narrow technocentric approach, as it failed to address the social causes of disease. Thus, it is considered as vertical program. Ideally, SPHC should be complementary to PHC, with specific and measurable target and effects. An active health workforce can be developed with adequate numbers of physicians, nurses, community health workers, and also with the allied health professions. Multisectorial approach like education, agriculture, housing, public works, rural development-all are equally important for promotion of health service in the developing countries.

Political commitment is a very important factor for implication of PHC. It can help as a link between health and development. As PHC is too broad based, most of the fund agencies are more interested to favor SPHC, which create difficulties for the government to provide health care to all levels of community.

As there is increasing aged population and consequent rise of noncommunicable diseases, PHC is taking steps to deal with these burden. WHO suggested adopting strategies to promote health, and to prevent and manage diseases in community within the prevailing health care system. Another important issue is mental health care, as this is closely associated with physical illness. PHC is involved in detection, diagnosis and treatment of common mental health problems in some local clinics, and referring the complicated cases to appropriate levels.

PHC model should have a single entry point to all kind of health and personal social services. This change would require a major investment in human resources, physical infrastructure, and information and communication technology [6]. Implementation of this model should be done on a phased basis. Refinement and evaluation of this model is necessary for success and development of a wider network.

Health-related millennium development goals cannot be achieved,

*Corresponding author: Rukhsana Parvin, Assistant Professor, Department of Medicine, Enam Medical College \& Hospital, Savar, Dhaka, Bangladesh, E-mail: rukhsana_parvin@yahoo.com

Received January 01, 2013; Accepted January 02, 2013; Published January 07 2013

Citation: Parvin R (2013) Primary Health Care. J Gen Pract 1: e101. doi: 10.4172/2329-9126.1000e101

Copyright: (c) 2013 Goodsite M, et al. This is an open-access article distributed under the terms of the Creative Commons Attribution License, which permits unrestricted use, distribution, and reproduction in any medium, provided the original author and source are credited. 
if values, principles and approaches of primary health care are not properly addressed. For the effective functioning of health services, adequate delivery of primary care services is essential. Restructuring national budgets along with increased health and other fundamental social services, can pave the way for major gains in health and development.

\section{References}

1. World Health Organization (1978) Declaration of Alma Ata. International conference on PHC, Alma-Ata, USSR, 6-12 September.
2. http://en.wikipedia.org/wiki/Primary_health_care

3. Cueto $M(2004)$ The origins of primary health care and selective primary health care. Am J Public Health 94: 1864-1874.

4. Primary Health Care: key to delivering cost-effective - disease control priority project.

5. Rohde J, Cousens S, Chopra M, Tangcharoensathien V, Black R, et al. (2008) 30 years after Alma-Ata: has primary health care worked in countries? Lancet 372: 950-961.

6. Primary Care-Executive Summary. 